\title{
Ear, Nose and Throat Emergencies and Anesthesia
}

\author{
${ }^{1}$ Minal Harde, ${ }^{2}$ Tushar Bawankar, ${ }^{3}$ Rakesh Bhadade
}

\begin{abstract}
Ear, nose and throat (ENT) emergency procedures demand significant anesthetic challenges. Variety of emergency conditions like deep neck space infections (Ludwig's angina, retropharyngeal, parapharyngeal abscess), acute onset of stridor, epiglottitis, laryngotracheobronchitis, inhaled foreign bodies, facial injuries, etc. are a challenge to both the surgeon and the anesthesiologist and communication and cooperation is vital. Priority in emergency is to clear and secure the airway and consider requirement of 'shared airway'. Preferred anesthesia and airway technique include awake flexible fiberoptic (FOB) guided intubation in cooperative patients' and an inhalational induction in uncooperative patient or tracheostomy. Anticipation, vigilance, readiness for difficult intubation, emergency tracheostomy and team work is essential while managing ENT emergency procedures.
\end{abstract}

Keywords: Difficult intubation, ENT emergency, Shared airway.

How to cite this article: Harde M, Bawankar T, Bhadade R. Ear, Nose and Throat Emergencies and Anesthesia. Int J Otorhinolaryngol Clin 2015;7(1):28-34.

Source of support: Nil

Conflict of interest: None

\section{INTRODUCTION}

Ear, nose and throat (ENT) emergency operations entail several unique anesthetic considerations. Provision of a clear, free, and unobstructed airway is the main concern for all ENT emergency procedures. Patients can present with various conditions to an emergency department including deep neck space infections (Ludwig's angina, retropharyngeal, parapharyngeal abscess), acute onset of stridor, epiglottitis, laryngotracheobronchitis, inhaled foreign bodies, facial injuries, etc. Diagnosis and management of above conditions is challenging and the priority is to clear and secure the airway. It is doubtless that the requirement for understanding between the surgeon and the anesthesiologist is greatest in ENT emergencies in

\footnotetext{
${ }^{1}$ Associate Professor, ${ }^{2,3}$ Assistant Professor

1,2Department of Anesthesiology, TN Medical College and BYL Nair Hospital, Mumbai, Maharashtra, India

${ }^{3}$ Department of Medicine, TN Medical College and BYL Nair Hospital, Mumbai, Maharashtra, India

Corresponding Author: Minal Harde, Associate Professor Department of Anesthesiology, TN Medical College and BYL Nair Hospital, Mumbai, Maharashtra, India, Phone: 0910222307137 e-mail: minalharde@yahoo.co.in
}

view of 'shared airway'. Anticipation, vigilance, readiness for difficult intubation, emergency tracheostomy and experienced anesthesiologist goes a long way in managing such emergencies.

\section{LUDWIG'S ANGINA}

Ludwig's angina is a severe diffuse cellulitis which spreads rapidly and becomes a potentially life-threatening condition due to the risk of impending airway obstruction. It is a medical emergency because of tendency to cause edema, distortion, and obstruction of the airway. Hence, early diagnosis, securing airway and treatment of Ludwig's angina is exceptionally important.

Ludwig's angina was first described in 1836 by Wilhelm Friedrich von Ludwig and angina implies the suffocating sensation. ${ }^{1}$ It is characterized as a rapidly progressive gangrenous cellulitis of the soft tissues of the neck and floor of the mouth. Primary cause is related to gingival or dental disease viz; infections of the second and third molars. Other causes include peritonsillar and parapharyngeal abscess, oral laceration, otitis media, lymphangiomas and mandibular fractures. ${ }^{2,3}$ The most commonly cultured organisms include Staphylococcus, Streptococcus and Bacteroides species. Atypical organisms isolated in these patients include Pseudomonas, Escherichia coli, Klebsiella, Enterococcus faecalis, Candida and Clostridium. Patients with immunocompromising conditions, such as HIV, diabetes, transplant recipients, and alcoholics, are at risk for infection from a variety of atypical organisms. ${ }^{4,5}$

Understanding the anatomy and pathogenesis of neck spaces and fascial planes is essential for effective treatment of this disease. The submandibular space is potential space above hyoid bone, which is divided into sublingual superiorly and submandibular spaces below by mylohoid muscle, these spaces behave as a one unit as they communicate posteriorly. The superficial layer of deep cervical fascia act as barrier along with mandible and hyoid bone for spread of infection. In effect, the path of least resistance toward airway becomes the most vulnerable area anatomically. ${ }^{2-5}$

Ludwig's angina is a clinical diagnosis. The majority of patients report dental pain, or a history of recent dental procedures and neck swelling. Less common complaints include neck pain, dysphonia, dysphagia, and dysarthria. Less than one third of adults will present in respiratory 
distress with dyspnea, tachypnea, or stridor. On physical examination, over $95 \%$ of patients have bilateral submandibular swelling and an elevated or protruding tongue. The submandibular swelling is often characterized as brawny and tense, with overlying erythema. In severe cases fatal asphyxia may occur. Acute loss of airway during interventions has also been reported as common cause of death and hence warrants extreme vigilance. ${ }^{6}$

\section{Management}

Airway control, antibacterial therapy and incision and drainage are the main stay of initial treatment. ${ }^{1-8}$ Airway management is the foundation of treatment for patients with Ludwig's angina. Antibiotics should be initiated as soon as possible. Antibiotics should initially be broadspectrum and cover Gram-positive, Gram-negative, and anaerobic organisms. Combinations of penicillin, clindamycin, and metronidazole are typically used.

\section{Anesthesia Implications}

Patients with Ludwig's angina often present with trismus due irritation of masticatory muscle, dyspnea, tachypnea, inspiratory stridor and cyanosis are signs of impending airway obstruction. The decision to secure the airway continues to rely on clinical judgment, review of diagnostic and imaging studies surgical plan and skill and experience. ${ }^{1,6}$

Difficult airway management equipment with tracheostomy should be kept ready. Endotracheal intubation is difficult in this patient may precipitate acute airway collapse hence awake fiberoptic intubation is method of choice in establishing the airway and should be first line of management. ${ }^{1}$

To reduce upper airway edema intravenous dexamethasone and nebulized epinephrine can be used. Orotracheal intubation by direct laryngoscopy may be difficult due to distorted airway anatomy. Blind nasal intubation is to be avoided as it could cause catastrophic bleeding, laryngospasm, airway edema, rupture of pus into the oral cavity and aspiration. ${ }^{7}$ Moreland et al in a literature review mentioned airway management protocol for these cases. In severely compromised airway patients (saturation on room below $95 \%$, respiratory rate $>25$, or a significant airway compromise on fiberoptic laryngoscopy) a definitive airway is required and awake, fiberoptic-assisted intubation should be attempted first; if this fails then a surgical tracheostomy is performed under local anesthesia. ${ }^{8}$

In skilled and experienced hands, flexible fiberoptic (FOB) nasal intubation is the preferred method of airway management and has a high rate of success. Tracheal intubation with a flexible bronchoscope by using topi- cal anesthesia is highly successful in adult patients with deep neck infections. In case of severe swelling or in the presence of pus and copious secretion where fiberoptic intubation is not possible or when not available, or has failed, tracheostomy is the option. ${ }^{6}$

Ludwig's angina is a life-threatening medical emergency. A team approach with anticipation and preparedness along with skill and experience is essential for successful management.

\section{STRIDOR}

Stridor is a harsh, noisy, vibratory high-pitched, predominantly inspiratory sound produced due to upper airway obstruction resulting in turbulent airflow in the respiratory passages. It is symptomatic of underlying pathology and may herald life-threatening airway obstruction. ${ }^{1}$ This can progress rapidly to complete airway obstruction and death from asphyxiation. Stridor at rest, implies that there is a reduction in airway diameter of at least $50 \%{ }^{3}$

The causes of stridor include the following:

- Infection: Peritonsillar abscess, retropharyngealabscess/ cellulitis, other deep neck abscesses, epiglottitis.

- Allergic: Angioneurotic edema

- Burns

- Foreign bodies

- Trauma

- Postsurgical: Procedures of the neck or upper airway, bilateral vocal cord palsies after surgery.

- Prolonged endotracheal intubation.

- Malignancy: Tumors of the upper airway or adjacent structures.

- Congenital abnormalities of the upper airway. Patient usually presents with abnormal respiratory pattern, harsh, noisy, high-pitched, inspiratory sound associated hypoxia or cyanosis are signs of impending complete upper airway obstruction. The presence of acute stridor in an alarming sign, its absence does not mean the airway is not compromised as patient may be becoming fatigued and a decrease in stridor may indicate impending respiratory arrest. When a patient progresses to complete airway obstruction, stridor and noisy breathing may cease because there is no further air movement. ${ }^{9}$

\section{Management and Anesthesia Considerations}

The initial management is symptomatic with $100 \%$ oxygen administered by a facemask or nasal prong in the sitting upright position. Establish IV access and monitoring pulse rate, ECG, NIBP, $\mathrm{SpO}_{2} \cdot{ }^{9,10}$

To reduce airway edema, nebulization with racemic epinephrine, $1 \mathrm{mg}$ of a $1: 1000$ solution $(1 \mathrm{ml})$ in $5 \mathrm{ml}$ of $0.9 \%$ saline and Dexamethasone, $0.1 \mathrm{mg} / \mathrm{kg}$ IV. Heliox (70\% helium, 30\% oxygen) increases air flow by decrease in airway resistance and improvement in ventilation. ${ }^{4,6,10}$ 
Investigation done to establish a firm diagnosis; however this should never delay definitive airway management. Routine blood investigations like complete blood count, blood grouping, blood sugar, etc. radiograph of the neck particularly for suspected foreign bodies or retropharyngeal abscess. In patients with stable airways CT scan should be done. ${ }^{9}$

Fiberoptic nasal endoscopy can be performed with nasal preparation using lidocaine and epinephrine. If an adequate view of the larynx cannot be obtained, problem with tracheal intubation is expected. However, successful visualization of the larynx with the patient awake and in the sitting position does not mean that it will be the same in a supine, anesthetized patient. ${ }^{11}$

Hence, always be prepared with difficult airway cart and FOB and a surgical airway. In advanced cases of perilaryngeal obstruction, the two main options are tracheostomy under local anesthesia or inhalational induction of anesthesia. While inducing patient with inhalational anesthesia (sevoflurane is preferred agent) after loss of consciousness respiratory obstruction may occur, hence nasopharyngeal airway after proper lubrication should be inserted. Oral airways are generally contraindicated as obstruction is not due posterior displacement of tongue but due to approximation of soft palate to posterior pharyngeal wall. ${ }^{10,11}$

Once the patient is deep enough, gentle laryngoscopy should be attempted, using a long or McCoy blade. Under direct vision decision must be made as to whether intubate or not. If, the anatomy is difficult to visualize, or the aperture is too small, it may be wise to withdraw and allow the surgeon to do tracheostomy while the patient still maintains adequate spontaneous respiration. ${ }^{11}$

Those patients with severe stridor, a large tumor, fixed hemilarynx, gross anatomical distortion or a larynx not visible on nasal endoscopy should undergo tracheostomy under local anesthesia without any sedation. Fiberoptic intubation not advised in patients with advanced obstruction from a periglottic tumor. ${ }^{11}$

\section{LARYNGOSPASM}

Laryngospasm is a medical emergency commonly encountered in children undergoing ENT procedures and needs urgent response.

Laryngospasm is an exaggeration of the normal glottic closure reflex (mediated by superior laryngeal nerve) which persists even after removal of the stimulus. Usually in the presence of a lighter plane of anesthesia upper airway or nasal irritation by blood, secretions, or surgical debris or painful, surgical stimulus results in exaggerated glottic closure reflex leading to laryngospasm.

Laryngospasm is very common after upper airway and ENT procedures in which blood, secretions, and surgical debris are present. Other causes of postextubation laryngospasm are laryngomalacia, tracheomalacia, vocal cord paralysis, airway edema, hematoma, soft tissue obstruction, and the presence of foreign material such as throat packs.

The incidence of laryngospasm is more in children than in adults, and hypoxia occurs rapidly. Children with airway hyperreactivity or with recent upper respiratory infections are more prone to develop laryngospasm.

\section{Management}

Preventive strategies for post-extubation laryngospasm in high risk children include administering IV lidocaine, topical lidocaine, IV magnesium and 'deep' extubation. ${ }^{10-14}$

Treatment starts with immediate suctioning to remove obvious blood and secretions from the airway and administering $100 \%$ oxygen with tightly held facemask. It acts as a pneumatic splint with either positive end-expiratory pressure or continuous positive airway pressure to keep the airway open. Larson's maneuver can be done which includes a forcible jaw thrust with bilateral digital pressure on the body of the mandible just anterior to the mastoid process, known as Larson's point, may resolve laryngospasm by clearing the airway and stimulation. ${ }^{10,13,14}$

If above maneuvers fail to break the laryngospasm, and oxygen saturation starts falling then increase the depth of anesthesia to diminish the exaggerated glottic closure reflex. It is preferably done with intravenous agents like propofol or ketamine. If, despite all the above it still persists and oxygen desaturation continues, a small dose of succinylcholine $(0.1-0.5 \mathrm{mg} / \mathrm{kg})$ should be administered.

\section{EPIGLOTTITIS}

Acute epiglottitis is an acute inflammation of the epiglottis, arytenoids, and aryepiglottic folds with rapid onset and progression leading to complete airway obstruction, hypoxemia and death. These are predicted difficult airways and an experienced anesthesiologist and ENT surgeon can save the patient from this fatal disease. Indiscriminate use of antibiotics in childhood results in failure to develop immunity against Haemophilus influenzae and hence the incidence is on the rise. ${ }^{10,15}$

Clinical presentation includes fever, sore throat, dysphagia, drooling, open mouth, muffled voice and respiratory distress. Stridor and tripoding are clues for impending sudden obstruction.

\section{Management and Anesthesia Considerations}

In adults, very careful flexible fiberoptic laryngoscopy (FFL), intravenous lines, and careful oropharyngeal 
examination can be done. However, any stimulation in children may precipitate complete airway obstruction so should be avoided.

If an X-ray can be done, 'thumbprint sign' (tissue swelling around hyoid bone) and the 'vallecula' sign (at the tongue base) are diagnostic of epiglottitis.

Difficult intubation preparation and emergency tracheostomy should be ready.

In children inhalational induction with sevoflurane in oxygen is preferred and done with the child usually in sitting position. Once the child is under anesthesia monitoring and IV access is established and anesthesia deepened with other IV agents. IV atropine and adequate IV fluids are must and nitrous oxide should be avoided. In adults, both inhalational and intravenous induction management can be used and follows similar principles.

On direct laryngoscopy, the epiglottis and aryepiglottic folds are swollen ('cherry-red' epiglottis) and the glottic opening may not be visualized. A small mucous bubble seen during spontaneous ventilation or by gentle pressure on the child's chest can be taken as a guide for intubation. ${ }^{10,15}$

A smaller, uncuffed oral endotracheal tube is preferred initially. Once the child is stable, nasotracheal tube is preferred for subsequent of ventilatory management. ${ }^{10,14,15}$ Antibiotic therapy according to throat and blood cultures is started and the child is managed in a pediatric intensive care unit.

\section{FACIAL NERVE COMPRESSION}

Facial nerve is mixed nerve having motor and sensory root. Labyrinthine segment is the shortest segment and bony canal also has the smallest diameter. In procedures, such as stapedectomy and mastoidectomy, facial nerve edema or inflammation can easily compress the nerve and cause facial paralysis.

Hence, facial nerve monitoring is indicated during middle ear, mastoid, and inner ear procedures to identify the facial nerve and reduce the incidence of iatrogenic facial nerve injury. Monitors have an audible and visible signal to identify when surgical movement or stimulation is close to, or at, the facial nerve. With neuromuscular blockade due to muscle relaxants this nerve activity is abolished and accidental transection of the facial nerve may occur. Hence, it is essential that neuromuscular blockade be stopped or reversed and an adequate depth of anesthesia can be maintained using remifentanil.

Sometimes nerve is paralyzed due to pressure of packing on exposed nerve which should be relieved first. Medical management of Bell's palsy includes treatment in the form of prednisolone $1 \mathrm{mg} / \mathrm{kg}$ / day in divided doses. Surgical management includes nerve decompression, cable nerve graft, reanastomosis of cut ends. Proper documentation, reassurance and follow-up is essential.

\section{FOREIGN-BODY AIRWAY OBSTRUCTION (CHOKING)}

Foreign-body airway obstruction (FBAO) in children $<5$ years of age is one of the commonest and potentially treatable cause of cardiac arrest. Sudden onset of respiratory distress with coughing, gagging, stridor, or wheezing in the absence of fever suggests FBAO.$^{16}$ Key to successful outcome is early recognition as signs are obvious but often fatal if undiagnosed and untreated.

Gustav Killian, a German otolaryngologist, performed the first bronchoscopy using a rigid esophagoscope to successfully remove a pig bone from a farmer's right main bronchus. ${ }^{17,18}$ Chevalier Jackson revolutionized endoscopic foreign body removal in the early 1900s with principles and techniques still followed today. ${ }^{17}$

\section{Etiology}

Foreign-body commonly occurs in children below the age of 15, more commonly in age group of 1 to 3 years. As they have tendency to put everything in the mouth while playing or running, inability to chew the food properly and also they lack coordination in swallowing and glottis closure.

Organic FB accounts for the $80 \%$ of cases while nonorganic causes rest of the cases. Peanuts are the most commonly encountered organic foreign body while pins are the most common nonorganic FB. Right bronchus is more commonly involved than left as it is more straight and broad. Laryngeal or tracheal FBs are rare but pose the greatest risk of asphyxia and carry a mortality rate of $45 \%$ prior to reaching a medical facility. ${ }^{16,19}$

\section{Clinical Presentation}

At times it is very difficult to diagnose the patient with aspirated foreign body as it is generally not witnessed and children usually do not give proper history. Therefore, the clinical presentation depends upon the size and shape of object inhaled, site of object within the airway (trachea/ main bronchus/distal airways) and time of presentation since event.

Foreign-body airway obstruction can be partial and presents with wheezing, cough and stridor or complete which presents with inability to speak, breathe or cough. Child Clutches neck and turns blue (Universal chocking sign). ${ }^{16}$

Laryngeal foreign bodies present with airway obstruction and hoarseness or aphonia. Tracheal foreign bodies present similarly to laryngeal foreign bodies and 
may demonstrate wheezing similar to asthma. Bronchial foreign bodies typically present with cough, unilateral wheezing, and decreased breath sounds.

A late presentation may well include signs of pneumonia, such as fever, cough, tachycardia, tachypnea and focal chest signs. However, none of the features above are $100 \%$ specific.

\section{Emergency Management of FBAO}

If coughing is ineffective, back blows, chest thrusts or abdominal thrusts (Heimlich maneuver) for children and adults can be used to try to expel the foreign body. These actions create an 'artificial cough' to increase intrathoracic pressure and dislodge the foreign body. ${ }^{16}$

Chest thrusts are used instead of Heimlich maneuver in infants and children up to 8 years as abdominal thrusts may damage the infant's relatively large and unprotected liver. Give up to 5 upward chest thrusts with a fist of one hand positioned over the lower half of sternum.

Back blows used in infant where 5 back blows delivered forcefully between shoulder blades with heel of hand ensuring an open mouth with prone, head low position. If the child becomes unresponsive, immediately basic life supports is initiated and start CPR with chest compressions. If foreign body is visible, remove it but do not perform blind finger sweeps. Attempt to give 2 breaths and continue with cycles of chest compressions and ventilations until the object is expelled. ${ }^{16}$

Once the airway is secured, or in non-asphyxiating cases, definitive treatment should be provided.

Definitive management: The gold standard method and preferred technique by the majority of surgeons for the removal of airway FBs is rigid bronchoscopy. This is largely because of the advantages a rigid bronchoscope provides in these cases namely, availability of multiple extraction instruments, good visualization, complete airway and FB control and an excellent conduit for ventilation. ${ }^{17}$ Broadly, the bronchoscope is divided into ventilating which has side port for ventilation to which $\mathrm{T}$-piece can be attached and the other is non-ventilating.

\section{Anesthesia Management}

The anesthetic management of foreign body aspiration is challenging. Good communication about sharing the airway with the operating surgeon is of paramount importance during the procedure. Detailed history and through preoperative check is essential as exact location, nature and duration of FB will decide further management. Laryngeal, tracheal and carinal FBs have the potential to cause complete airway obstruction therefore present the highest risk for inadequate oxygenation. Danger signs would include hypoxia on presentation, stridor, voice changes and dysphonia or aphonia. 19,20
Esophageal FBs are more commonly encountered than airway FBs and morbidity and mortality related to their removal is considerably less as the airway is not shared. $^{20,21}$

Organic FB has potency to absorb fluid and swell causing progressive airway obstruction and is also prone for fragmentation. ${ }^{22}$ Nuts and seeds liberate irritant oils causing airway inflammation and irritability with the risk of bronchospasm. Spherical shaped FBs are prone to complete obstruction of bronchial airways because of their shape which can lead to distal atelectasis with distal suppuration and possible spillage of pus into unaffected parts of the lung once it is removed. ${ }^{19-22}$

Duration of aspiration helps in knowing the status of patient as if patient with delayed presentation are generally associated with infection, airway edema and formation of granulation tissue around FB which make its retrieval difficult and prolong the anesthesia time.

Recently aspirated FBs are more likely to move when the patient coughs and can potentially lodge in the upper airway and can lead to complete obstruction.

\section{Investigation}

$X$-ray: Anteroposterior and lateral chest $X$-ray along with neck are done using high kilo voltage (KV) so that airways are better visualized than bony structures. Chest $X$-ray also helps in diagnosis of atelectasis, consolidation, emphysema, and gas trapping (ball valve mechanism). Lateral decubitus is mainly used in children with bronchial foreign body showing hyperinflation. Airway fluoroscopy allows dynamic visualization of respiratory airways. It can display decreased diaphragm movements due to air trapping, narrowed airway lumen from a FB and mediastinal shift from air trapping. As most of the foreign bodies are organic in nature may be not visible on chest X-ray till 24 hours. Hence, it should not exclude the diagnosis if not visible on $X$-ray. ${ }^{20}$ Computed tomography thorax and virtual bronchoscopy are more sensitive.

Rigid bronchoscopy: It is considered as the gold standard for diagnosis and management of suspected airway FBs it can be used both as diagnostic as well therapeutic in some case, but it carries high rate of complication. Hence, it is recommended to use rigid bronchoscopy only when there is clear history of asphyxiation, radio-opaque FB, unilateral pulmonary signs and obstructive emphysema. Rest of the patient should undergo flexible bronchoscopy.

\section{Premedication}

Primary aim of premedication to keep the child calm and prevent coughing as it can lead to dislodgement and further airway obstruction. Sedative medications are generally avoided as its decrease the respiratory drive 
which will further worsen the obstruction. Hence, utmost care should be taken while given sedation in agitated patient. Anticholinergic helps to reduce secretions and reflex bradycardia associated with airway instrumentation. Antibiotic and steroid medications are indicated specially in delayed presentations where infection and laryngeal edema is present and bronchoscopic manipulation is expected to be excessive. ${ }^{10,22-25}$

\section{Intraoperative Considerations}

The main goals of anesthesia for rigid bronchoscopy are oxygenation and immobile patient. Various successful anesthetic techniques are described for airway FB removal. Prior to induction it is very important that the surgeon to be in theater with the immediate ability to perform rigid bronchoscopy if total airway obstruction occurs. A minimum of two anesthetists (one for drug administration and the other for airway management) is critical to a successful outcome.

Close observation and monitoring throughout the procedure is required to ensure a correct depth of anesthesia to prevent movement, coughing and laryngospasm. ${ }^{10}$

Both intravenous (IV) and inhalational induction are used with successful outcomes. The choice between IV and inhalational induction can be decided by the location of the FB. Proximal airway FBs (larynx, trachea and carina) have the ability to move and completely obstruct the airway once spontaneous ventilation (SV) is converted to positive pressure ventilation (PPV) as may occur after IV induction. For this reason inhalational induction is advocated when proximal airway FBs are present. ${ }^{22}$

Inhalational induction is preferred by most anesthetists for the removal of FBs in children. Advantages include less irritant in pediatric patients, high success rate with maintaining SV, hemodynamic stability and the ability to titrate and quickly reverse. After induction but prior to bronchoscopy use topical local anesthesia with lignocaine $1 \%$ (maximum safe dose is $4 \mathrm{mg} / \mathrm{kg}$ ) sprayed onto the larynx and into the trachea to reduce the cardiovascular and tussive response to bronchoscopy. ${ }^{10,22-25}$

Both SV and PPV has been used successfully by various authors and there are no significant difference in major outcomes like mortality. ${ }^{22}$ Spontaneous ventilation has the advantage of continued ventilation and oxygenation despite interruptions with the anesthetic breathing circuit. But in cases of high airway resistance due to lung (like pneumonia) there is increase work of breathing and can lead to desaturation. The major disadvantage of SV is the high incidence of coughing and / or bucking during bronchoscopy because of inadequate depth of anesthesia hence there is high risk of trauma and rupture of airway while manipulation.
Spontaneous ventilation around the bronchoscope is more suitable for removal of proximal FB, during which leakage around the scope may make effective PPV difficult. Manually closing the mouth and nose can diminish a large leak around the scope and improve ventilation. However, these may interfere with surgeon's field.

Many bronchoscopes have the attachment of a T-piece to a side arm through which oxygen and volatile agent can pass directly into the distal trachea. Positive pressure ventilation down the bronchoscope with intermittent apnea while manipulating the object may be more suitable for distal retrieval. But there is a risk of PPV causing distal air trapping by a ball-valve effect. ${ }^{22-25}$

An alternative solution to this problem is the use of jet ventilation. Manual jet ventilation via a side port of the bronchoscope is described and was shown to have fewer episodes of hypoxemia compared to PPV with a T-piece during FB retrieval. Its use in children is not advocated, as barotrauma is a common complication. ${ }^{26}$

For maintenance of anesthesia sevoflurane is preferred, with the advantage of relative stability of the cardiovascular system and quick control of depth. With availability of ventilating bronchoscope maintenance with inhalation agent is quite comfortable. Total intravenous anesthesia (TIVA) is also practiced as it provides a constant depth of anesthesia irrespective of ventilation mode (SV or PPV), and also during manipulation of the bronchoscope in and out of the airways. Commonly used drug are propofol, remifentanyl, dexmedetomidine and midazolam. Dosages in the range of $0.2 \mu \mathrm{g} / \mathrm{kg} / \mathrm{min}$ of remifentanil and 100 to $250 \mu \mathrm{g} / \mathrm{kg} / \mathrm{min}$ of propofol provide adequate surgical conditions. ${ }^{22-28}$

Perioperative complications encountered are complete airway obstruction (secondary to dislodged FB during coughing or removal), failure to remove FB, hypoxia, hypercarbia, coughing, regurgitation and aspiration of gastric contents, pneumothorax, pneumonia laryngeal edema. Cardiac dysrhythmias, and cardiac arrest may also occur.

In the postoperative period significant pulmonary compromise (hypoxemia) and upper airway obstruction from laryngeal edema should be anticipated after FB removal, especially in delayed presentations where bronchoscopy was difficult and prolonged hence warrants high-dependency postoperative care. Nursing the child with the affected lung down can help prevent contamination of the unaffected lung. Supplemental humidified oxygen, adrenalin saline nebulization, intravenous steroids (dexamethasone, $0.1 \mathrm{mg} / \mathrm{kg}$ ) and antibiotics should be continued. ${ }^{10,23}$ 


\section{SUMMARY}

Of all patient groups, ENT emergency patients have the highest likelihood of having difficult airway. If the airway is suspicious then awake FOB guided intubation in cooperative patients' and an inhalational induction in uncooperative patient are preferred. Tracheostomy should be absolutely ready. If there is a chance of postoperative edema that may obstruct the airway (e.g. tongue), or suspicion of laryngotracheomalacia the patient should be electively left intubated and carefully observed in high dependency unit.

Thus, exquisite cooperation between the anesthesiologist and surgeon, careful planning, preoperative evaluation, anticipation, extreme vigilance and preparedness is essential while managing ENT emergency procedures.

\section{REFERENCES}

1. Kulkarni AH, Pai SD, Bhattarai B, Rao ST, Ambareesha M. Ludwig's angina and airway considerations: a case report. Cases J 2008;1:19.

2. Balasubramanian $S$, Elavenil $P$, Shanmugasundaram $S$, Himarani J, Krishnakumar Raja VB. Ludwig's angina: a case report and review of management. SRM J Res Dent Sci 2014; 5:211-214

3. Candamourty R, Venkatachalam S, Babu MR, Kumar GS. Ludwig's Angina-an emergency: a case report with literature review. J Nat Sci Biol Med 2012;3:206-208.

4. Winters M. Evidence-based diagnosis and management of ENT emergencies. AANA J 2006;74:6.

5. Kassam K, Messiha A, Heliotis M. Ludwig's Angina: the original angina. Case Rep Surg 2013; 2013:974269.

6. Ovassapian A, Tuncbilek M, Weitzel EK, Joshi CW. Airway management in adult patients with deep neck infections: a case series and review of the literature. Anesth Analg 2005;100: 585-589.

7. Duprey K, Rose J, Fromm C. Ludwig's angina. Int J Emerg Med 2010;3:201-202.

8. Moreland LW, Corey J, McKenzie R. Ludwig's angina. Report of a case and review of the Literature. Arch Intern Med 1988;148:461-466.

9. Maloney E. Meakin GH. Acute stridor in children. Contin Educ Anaesth Crit Care Pain 2007;7(6):183-186.

10. Rees L, Mason RA. Advanced upper airway obstruction in ENT surgery. Br J Anaesth (CEPD Reviews) 2002;2:134-137.

11. Miller RD. Anesthesia for Eye, Ear, Nose, and Throat Surgery. Marc Allan Feldman and Anil Patel editors. Millers Anesthesia. 7th ed. Young Elsevier Health 2010;2357-2388.

12. Ernst A, Feller-Kopman D, Becker HD, Mehta AC. Central airway obstruction. Am J Respir Crit Care Med 2004; 169:12781297.

13. Gulhas N, Durmus M, Demirbilek S, et al. The use of magnesium to prevent laryngospasm after tonsillectomy and adenoidectomy: a preliminary study. Pediatr Anesth 2003; 13:43-47.

14. Gray H. Extubation. In: Calder I, Pearce A, editors: core topics in airway management. Cambridge, Cambridge University Press, 2005:87-92.

15. Abdallah C. Acute epiglottitis: trends, diagnosis and management. Saudi J Anaesth 2012;6:279-281.

16. Berg MD, Schexnayder SM, Chameides L, Terry M, Donoghue A, Hickey RW, Berg RA, Sutton RM, Hazinski MF. Part 13: pediatric basic life support: 2010 American Heart Association Guidelines for Cardiopulmonary Resuscitation and Emergency Cardiovascular Care. Circulation 2010;122(suppl 3): S862-S875.1.

17. Fidkowski CW, Zheng H, Firth PG. The anesthetic considerations of tracheobronchial foreign bodies in children: a literature review of 12,979 cases. Anesth Analg 2010;111(4): 1016-1025.

18. Clerf LH. Historical aspects of foreign bodies in the air and food passages. South Med J 1975;68:1449-1454.

19. Zur KB, Litman RS. Pediatric airway foreign body retrieval: surgical and anesthetic perspectives. Pediat Anesth 2009;19: 109-117.

20. Rodríguez H, Passali GC, Gregori D, Chinski A, Tiscornia C, Botto $\mathrm{H}$, et al. Management of foreign bodies in the airway and oesophagus. Int J Pediat Otorhinolaryngol 2012;76: S84-S91.

21. Zaytoun GM, Rouadi PW, Baki DH. Endoscopic management of foreign bodies in the tracheobronchial tree: predictive factors for complications. Otolaryngol Head Neck Surg 2000;123:311-363.

22. Farrell PT. Rigid bronchoscopy for foreign body removal: anaesthesia and ventilation. Pediat Anesth 2004;14(1):84-89.

23. Fidkowski CW, Zheng H, Firth PG. The anesthetic considerations of tracheobronchial foreign bodies in children: a literature review of 12,979 cases. Anesth-Analg 2010;(111):4.

24. Litman RS, Ponnuri J, Trogan I. Anesthesia for tracheal or bronchial foreign body removal in children: an analysis of ninety-four cases. Anesth Analg 2000;91:1389-1391.

25. Soodan A, Pawar D, Subramanium R. Anesthesia for removal of inhaled foreign bodies in children. Pediat Anaesth 2004; 14(11):947-952.

26. Li S, Liu Y, Tan F, Chen J, Chen L. Efficacy of manual jet ventilation using Manujet III for bronchoscopic airway foreign body removal in children. Int J Pediatric Otorhinolaryngol 2010;74(12):1401-1404.

27. Teksan L, Baris S, Karakaya D, Dilek A. A dose study of remifentanil in combination with propofol during tracheobronchial foreign body removal in children. J Clinical Anesth 2013;25(3):198-201.

28. Liao R, Li JY, Liu GY. Comparison of sevoflurane volatile induction/maintenance anaesthesia and propofol-remifentanil total intravenous anaesthesia for rigid bronchoscopy under spontaneous breathing for tracheal/bronchial foreign body removal in children. European J Anesthesiol 2010;27(11): 930-934. 